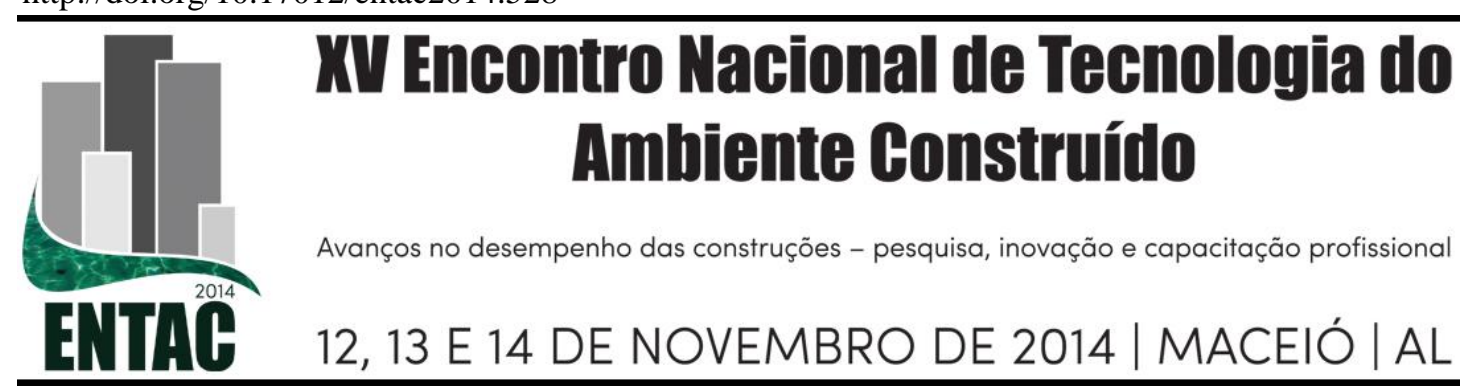

\title{
AVALIAÇÃO DA ADEQUAÇÃO DO GESSO RECICLADO ÀS PRESCRIÇÕES DA NBR 13207
}

\author{
MOURA, R. S. L. M. (1); DEUSDARA, D. F. M. (2); BARRETO, J. M. L. (3); \\ CABRAL, A. E. B. (4)
}

(1) UFC, e-mail: rafaelleall@hotmail.com, (2) UFC, e-mail: davideusdara@gmail.com, (3) UFC, e-mail: mauriciobarreto@ufc.br,(4)UFC, e-mail: eduardo.cabral@ufc.br

\begin{abstract}
RESUMO
Os resíduos gerados pela construção civil representam uma grande parcela dos resíduos sólidos urbanos, o que pode ser atribuído à alta intensidade de novas construções, bem como às elevadas taxas de desperdício de materiais nos canteiros de obras. Perdas de material na aplicação ainda são altas, tendo sido observados em obras de edifícios brasileiros valores medianos de $30 \%$. Para produzir o gesso, o minério gipsita é extraído de jazidas naturais, triturado, calcinado à temperatura de $150^{\circ} \mathrm{C}$ para perder umidade, sendo posteriormente moído e após hidratado, estará pronto para ser utilizado. Trabalhos científicos revelam que o descarte inadequado do gesso no meio ambiente é perigoso pois pode ser liberado o gás sulfídrico, irritante para as mucosas e causador de vários problemas respiratórios. $\mathrm{O}$ objetivo deste trabalho foi comparar as propriedades físicas, químicas e mecânicas do gesso comercial (GC), utilizado como referência, com as do gesso comercial reciclado (GCR) e resíduo de gesso reciclado (RGR), além da verificação da conformidade de todos com a NBR 13207 (ABNT, 1994), através de ensaios em laboratório. Foi feita uma análise do processo de reciclagem e verificado o que acarreta nas propriedades químicas, físicas e mecânicas do material reciclado. A análise das características dos gessos reciclados (GCR e RGR) com as características do gesso comercial utilizado como referência indica que seguindo estes métodos para reciclagem do gesso, o GCR e RGR não atendem satisfatoriamente nenhuma das exigências para classificação como gesso de construção civil pela NBR 13207 (ABNT, 1994), em relação às propriedades físicas e químicas do pó de gesso, físicas da pasta e mecânica do gesso.
\end{abstract}

Palavras-chave: Gesso, Resíduo da construção civil, Reciclagem.

\begin{abstract}
Waste generated by construction represents a large portion of municipal solid waste, which can be attributed to the high intensity of new buildings as well as the high rates of materials waste at construction sites. Material waste in the application still is high, it was observed in the works of Brazilian buildings median values of $30 \%$. To produce gypsum, the gypsum ore is mined from natural deposits, crushed, calcined at $150{ }^{\circ} \mathrm{C}$ to lose humidity, after being milled and after hydrated, it is ready to be used. Papers reveal the improper disposal of gypsum in the environment is dangerous because it can be released gas sulfide, it is irritating to mucous membranes and causes various respiratory problems. The aim of this study is to compare the physical, chemical and mechanical properties of Commercial Gypsum (GC) used like reference, the Recycled Business Gypsum (GCR) and Waste of Recycled Gypsum (RGR), and to check conformity of all them with the NBR 13207 (ABNT, 1994) through laboratory test. The analysis of the characteristics of recycled gypsum (GCR and RGR) with the characteristics of commercial gypsum indicates that following these methods for the recycling of gypsum, the GCR and RGR don't get satisfactorily any requirements for classification as plaster of the construction by NBR 13207 (ABNT, 1994), about the physical and chemical properties of the gypsum powder, physical of the paste and mechanical of the gypsum.
\end{abstract}

Keywords: Gypsum, Construction waste, Recycling. 


\section{INTRODUÇÃO}

Ao longo dos anos, a indústria da construção civil vem agregando para si parâmetros hoje imprescindíveis para um melhor desenvolvimento sustentável do planeta, aceitando o uso de produtos que sejam considerados ecologicamente corretos, reduzindo, portanto impactos no meio ambiente decorrente de resíduos gerados pela mesma. Uma preocupação da indústria é com a geração de resíduos e entulhos nas construções. Sendo assim, procurar também formas de reutilizá-lo é algo de vital importância para a sociedade. Em maio de 2011, a resolução $\mathrm{N}^{0} 431$ do CONAMA (BRASIL, 2011) definiu o gesso como classe $\mathrm{B}$ na classificação dos resíduos de construção e demolição (RCD) que o classifica como reciclável. Ou seja, a partir desta data os resíduos de gesso deverão agora serem reciclados.

A gipsita é o sulfato de cálcio hidratado com duas moléculas de água $\left(\mathrm{CaSO}_{4} \cdot 2 \mathrm{H}_{2} \mathrm{O}\right)$. Das duas moléculas de água, uma e meia está fracamente combinada e a outra meia molécula fortemente combinada. Isto explica o porquê da ocorrência de pelo menos duas fases distintas na fabricação de gesso. Na primeira fase se desprende a água fracamente combinada, é quando se obtém o hemidratado $\left(\mathrm{CaSO}_{4} \cdot 1 / 2 . \mathrm{H}_{2} \mathrm{O}\right)$, e a segunda fase quando se desprende a água fortemente combinada, obtendo-se o sulfato anidro solúvel $\left(\mathrm{CaSO}_{4}\right)(\mathrm{ROQUE}$ ET AL., 2005).

O Gesso de sulfatos hidratados e anidros de cálcio, é obtido pela calcinação (decomposição a quente) da gipsita natural à cerca de $160^{\circ} \mathrm{C}$ constituída de sulfato bi hidratado de cálcio geralmente acompanhado de certa proporção de impurezas, como sílica, alumina, óxido de ferro, carbonatos de cálcio e magnésio. O total das impurezas varia desde uma porção muito pequena até um máximo de cerca de $6 \%$ (BAUER, 2000).

A quantidade de gesso consumida pela população brasileira ainda é considerada baixa, $9 \mathrm{~kg} / \mathrm{hab}$. em 2003, calculado a partir do consumo aparente fornecido pelo Departamento Nacional de Produção Mineral e da população, pelo Instituto Brasileiro de Geografia e Estatística (AGOPYAN ET AL., 2005).

Considerando a necessidade de reciclar os resíduos gerados na cadeia produtiva do gesso e avaliar as propriedades do material reciclado para aplicação na construção civil este trabalho tem como objetivos comparar as propriedades físicas, químicas e mecânicas do gesso comercial (GC), utilizado como referência, com as do gesso comercial reciclado (GCR) e resíduo de gesso reciclado (RGR), verificando se estes estão em conformidade com a NBR 13207 (ABNT, 1994), através de ensaios em laboratório.

\section{REVISÃO BIBLIOGRÁFICA}

O gesso de construção é um material produzido por calcinação do minério natural gipso ou de sulfato de cálcio hidratado residual, constituído essencialmente de sulfatos de cálcio - hemidrato, anidridas solúvel e insolúvel - e a gipsita procedente de matéria prima. Como os gessos apresentam velocidade de pega e endurecimento muito mais rápida que o cimento Portland e a cal hidratada, em algumas variedades a ação se completa em menos de uma hora. Para haver maior flexibilidade na aplicação, é comum a sua utilização com aditivos retardadores (JOHN; CINCOTTO, 2010).

Conforme John e Cincotto (2010), o processo de produção do gesso de construção no Brasil é realizado, normalmente, a partir de matéria-prima natural, envolvendo as seguintes etapas: extração, britagem, moagem grossa e estocagem com 
homogeneização, em seguida secagem, calcinação, moagem fina e ensilagem ou embalagem.

A NBR 13207 (ABNT, 1994) classifica dois tipos de gesso em função da aplicação: em revestimento e em fundição, sendo que ambos têm como requisito para classificação o tempo de pega e o módulo de finura. As exigências mecânicas e químicas são as mesmas para todos os tipos de gesso.

É possível reciclar o gesso acartonado que contém outros compostos, produzindo aglomerantes, desde que sejam removidos contaminantes incorporados no processo de geração de resíduos (CAMPBELL, 2003). O gesso em contato com umidade e condições anaeróbicas, com baixo $\mathrm{pH}$, e sob ação de bactérias redutoras de sulfatos, condições presentes em muitos aterros sanitários e lixões, pode formar gás sulfídrico $\left(\mathrm{H}_{2} \mathrm{~S}\right)$, que possui odor característico de ovo podre, tóxico e inflamável (CIWMB, 2003).

A reciclagem dos resíduos de gesso é tecnicamente possível, com várias aplicações. Estudos nesse sentido foram desenvolvidos por Carvalho (2005) e Nita et al. (2004), cujos resultados preliminares evidenciam a viabilidade técnica de utilização do material reciclado na forma hemidratada. Todavia, o resíduo de gesso pode conter, além do sulfato de cálcio di-hidratado, teores de hemi-hidratos, anidritas e outros inertes (impurezas) que alteram sua composição química em relação à gipsita natural. Os cristais de di-hidrato do resíduo de gesso, devido às condições em que foram hidratados, podem apresentar morfologias diferenciadas da gipsita natural (JOHN; CINCOTTO, 2010).

Visando a produção de um gesso com características apropriadas à reciclagem, Cavalcanti (2006), Monção Júnior (2008) e Melo (2008) desenvolveram um processo de calcinação em que a gipsita calcinada à temperatura de $170^{\circ} \mathrm{C}$ sob pressão de $0,65 \mathrm{~atm}$ produz um gesso de construção, que ao ser hidratado gera resíduos cujas características proporcionam a produção de um gesso reciclado com características físicas e mecânicas melhoradas à reciclagem.

Niglio e Camarini (2004) estudaram em laboratório as propriedades do gesso reciclado proveniente dos resíduos de pasta de revestimento de obras, considerando as seguintes condições do processo de reciclagem: (I) moagem do resíduo com $80 \%$ da massa passante do material na peneira com abertura de malha de $0,297 \mathrm{~mm}$; (II) calcinação em estufa, nas temperaturas de $100^{\circ} \mathrm{C}, 160^{\circ} \mathrm{C}$ e $200^{\circ} \mathrm{C}$, com tempo de permanência de 24 horas. Os gessos reciclados, obtidos por este processo, apresentam as seguintes características, no material em pó e propriedades físicas no estado fresco (BARDELLA; CAMARINI, 2004, 2006): massa unitária do gesso reciclado é inferior à massa unitária do gesso comercial; módulo de finura maior que o do gesso comercial; consistência das pastas de gesso reciclado reduzida em todas as temperaturas estudadas; tempo de início de pega retardado, para as temperaturas de calcinação de $160^{\circ} \mathrm{C}$ e $200^{\circ} \mathrm{C}$.

Os autores concluem que o fator temperatura de calcinação influencia no tempo de pega do material reciclado. Todavia, alertam que o fator impureza do resíduo pode contribuir para essa alteração. Isso fica evidenciado com os valores encontrados por Niglio e Camarini (2004), pois, embora os resíduos sejam de mesma natureza, o tempo de início de pega é acelerado para o gesso reciclado à temperatura de $160^{\circ} \mathrm{C}$.

As propriedades mecânicas encontradas pelos autores mostram que o gesso reciclado pode alcançar os mesmos níveis do gesso comercial, apresentando melhor desempenho 
o gesso reciclado a $200^{\circ} \mathrm{C}$, possivelmente devido à redução da permeabilidade avaliada nesse material (BARDELA; CAMARINI, 2004; NIGLIO; CAMARINI, 2004).

\section{METODOLOGIA}

\subsection{Materiais}

O gesso comercial (GC) utilizado no trabalho como referência é comercializado em sacos de $1 \mathrm{~kg}$ e segundo classificação da NBR 13207 (ABNT, 1994) é gesso fino para fundição. As propriedades físicas e químicas do pó utilizado, propriedades físicas da pasta de gesso e propriedades mecânicas do gesso foram determinados em laboratório e encontram-se nas Tabelas 01 e 02 respectivamente.

Tabela 01 - Propriedades físicas e mecânicas

\begin{tabular}{cccc}
\hline Determinação & Unidade & Referências normativas & Resultados \\
\hline Módulo de finura & - & NBR 13.207/1994 & 0,14 \\
\hline Massa unitária & $\mathrm{kg} / \mathrm{m}^{3}$ & NBR 13.207/1994 & 729,87 \\
\hline Tempo de início de pega & $\mathrm{min}$ & NBR 13.207/1994 & $13: 25$ \\
\hline Tempo de fim de pega & $\mathrm{min}$ & NBR 13.207/1994 & $22: 17$ \\
\hline Dureza & $\mathrm{N} / \mathrm{mm}^{2}$ & NBR 13.207/1994 & 31,12 \\
\hline Resistência à compressão & $\mathrm{MPa}$ & NBR $13.207 / 1994$ & 8,00 \\
\hline
\end{tabular}

Fonte: Os autores

Tabela 02 - Composição química

\begin{tabular}{lc}
\hline Elemento & \% Massa \\
\hline $\mathrm{CaO}$ & 70,22 \\
\hline $\mathrm{SO}_{3}$ & 28,04 \\
\hline $\mathrm{SiO}_{2}$ & 0,56 \\
\hline $\mathrm{Sr}$ & 0,39 \\
\hline $\mathrm{Fe}_{2} \mathrm{O}_{3}$ & 0,38 \\
\hline $\mathrm{Al}_{2} \mathrm{O}_{3}$ & 0,26 \\
\hline $\mathrm{K}$ & 0,14 \\
\hline
\end{tabular}

Fonte: Os autores

O resíduo de gesso $(\mathrm{RG})$ utilizado para reciclagem foi coletado na Usina de Reciclagem do Nordeste (USINE) localizada na Estrada do Ancuri S/N - Distrito de Ancuri Itaitinga / CE. Foi coletada uma amostra aleatória em uma baia (Figura 01) na qual o resíduo é depositado.

Não foi feita a caracterização do resíduo de gesso $(R G)$, pois se trata de um material inerte, uma vez que já teriam ocorridas as reações de endurecimento do gesso (após reação apresentam características da pedra gipsita), as amostras para comparação seriam do resultado do processo de reciclagem, além de ter encontrado parâmetros para comparação das características de gesso endurecido. Porém é possível ver facilmente a contaminação do resíduo de gesso por outros materiais. 
Figura 1 - Baia com resíduo de gesso

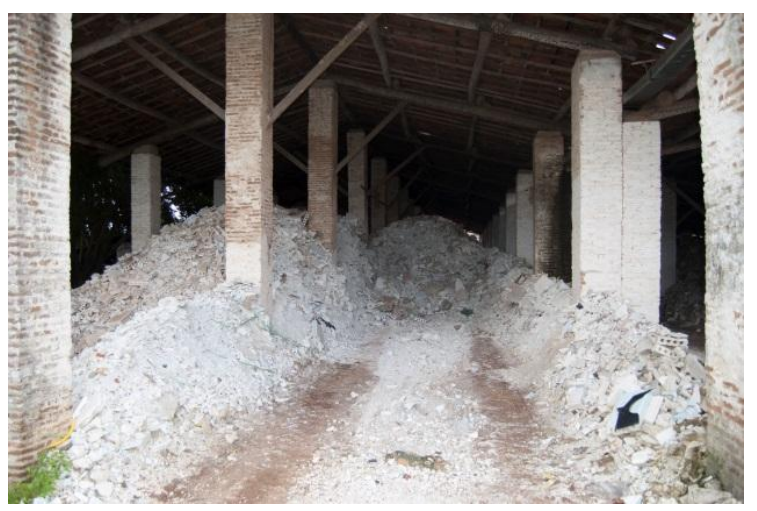

Fonte: Os autores

\subsection{Método de pesquisa}

Primeiramente os gessos, tanto $\mathrm{GC}$ endurecido quanto o $\mathrm{RG}$, foram quebrados em pedaços pequenos para serem triturados no britador de mandíbulas, ficando com diâmetro máximo de aproximadamente dois centímetros. Após a britagem foram passados pela peneira de malha de $4,8 \mathrm{~mm}$ para ficarem com granulometria semelhante antes da calcinação, tanto o $\mathrm{GC}$ endurecido quanto o RG, sendo separados em recipientes distintos e herméticos. Logo após, estas amostras foram dispostas em bandejas metálicas retangulares e calcinadas em estufa a uma temperatura constante de $150^{\circ} \mathrm{C}$ por um período de 24 horas.

Após uma análise expedita da granulometria do gesso de referência, percebeu-se que os grãos deste passavam com facilidade na peneira de malha de $0,6 \mathrm{~mm}$ e passavam com dificuldade na de malha de $0,425 \mathrm{~mm}$. Foi então separado apenas o material passante na peneira de malha de $0,6 \mathrm{~mm}$ depois de moído no moinho de bolas para uma comparação mais verossímil possível com o gesso de referência, simulando assim a moagem fina e peneiramento no processo de fabricação do gesso.

Para caracterizar o gesso de referência e os novos materiais foram realizados ensaios para determinação física e química do pó, física da pasta e mecânica do gesso endurecido. Foram determinadas a granulometria do pó, por meio da granulometria a laser, a massa unitária e a composição química do mesmo, por meio da fluorescência de raios-x (FRX). Determinou-se ainda o tempo de pega das pastas e a dureza e a resistência à compressão das pastas enrijecidas.

Nos ensaios em que os gessos foram hidratados, o valor da relação de massa água/gesso foi fixada em 0,50, apesar da NBR 12128 (ABNT, 1991) prescrever o método para execução do ensaio de consistência normal que determina a relação adequada na qual se obtém uma fluidez da pasta adequada à manipulação. Este procedimento foi seguido porque não foi possível estabelecer esta relação para o gesso originado da reciclagem do resíduo de gesso devido o seu endurecimento rápido, assim tal valor foi escolhido pela sugestão no rótulo da embalagem do gesso comercial.

\section{RESULTADOS}

Os resultados das propriedades físicas do pó de gesso estão apresentados na Tabela 03. 
Tabela 03 - Características físicas do pó de gesso

\begin{tabular}{ccc}
\hline Amostra & Módulo de finura & Massa unitária $\left(\mathbf{k g} / \mathbf{m}^{\mathbf{3}}\right)$ \\
\hline GC & 0,14 & 729,87 \\
\hline GCR & nd & 588,18 \\
\hline RGR & nd & 596,84 \\
\hline
\end{tabular}

Fonte: Os autores. nd - não determinado

Os resultados da granulometria a laser das amostras de gesso indicam que os grãos do RGR são mais grossos que os grãos do GC e GCR, sendo que estes últimos apresentaram valores bem próximos. Conforme apresentado na Tabela 04 pode-se observar que $90 \%$ dos grãos do GC têm diâmetro menor que $163 \mu \mathrm{m}$, no caso do GCR são menores que $270 \mu \mathrm{m}$ e no RGR $90 \%$ dos grãos passantes tem diâmetro menor que $446 \mu \mathrm{m}$. A área superficial específica do GC é $0,856 \mathrm{~m}^{2} / \mathrm{g}$, do GCR é $0,910 \mathrm{~m}^{2} / \mathrm{g}$ e do RGR $0,547 \mathrm{~m}^{2} / \mathrm{g}$, sugerindo que os grãos do GC e GCR são mais finos que os do RGR. O RGR apresentou grãos mais grossos possivelmente devido à dificuldade de moagem pela presença de impurezas, como grãos de areia que poderiam ter sido misturados nas baias de armazenamento.

Tabela 04 - Valores de d(0,1), d(0,5) e d(0,9)

\begin{tabular}{lccc}
\hline & GC & GCR & RGR \\
\hline Área superficial específica $\left(\mathbf{m}^{2} / \mathbf{g}\right)$ & 0,856 & 0,910 & 0,547 \\
\hline $\mathbf{d}(\mathbf{0 , 1})(\boldsymbol{\mu m})$ & 2,877 & 2,506 & 4,545 \\
\hline $\mathbf{d}(\mathbf{0 , 5})(\boldsymbol{\mu m})$ & 16,382 & 18,595 & 63,695 \\
\hline $\mathbf{d}(\mathbf{0 , 9})(\boldsymbol{\mu m})$ & 162,71 & 269,426 & 445,57 \\
\hline
\end{tabular}

Fonte: Os autores

Os resultados da massa unitária dos gessos estão apresentados no Gráfico 01. Pode-se observar que o GCR e o RGR apresentaram massa unitária inferior a dos demais e 16\% e $15 \%$ respectivamente, inferiores ao valor mínimo exigido pela NBR 13207. Este comportamento já era esperado pois usualmente os materiais reciclados apresentam massa unitária inferior a dos materiais virgens.

\section{Gráfico 01 - Massa unitária dos gessos}

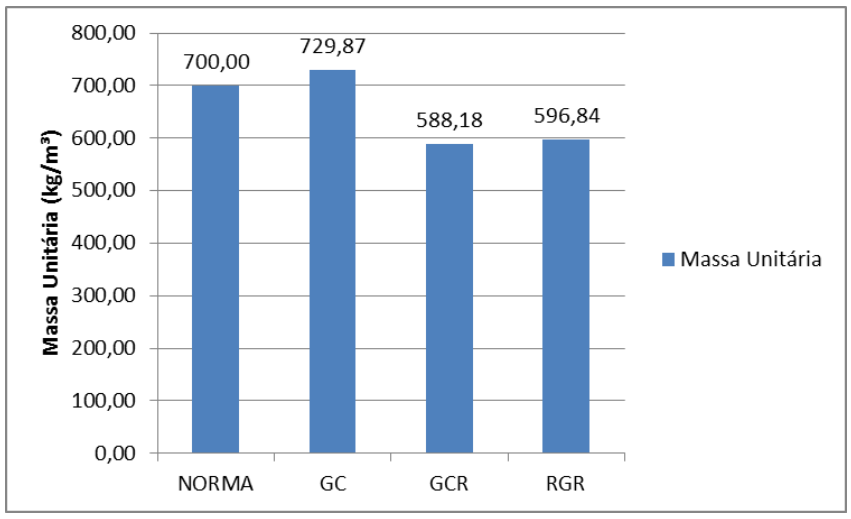

Fonte: Os autores 
A composição química dos gessos estudados, determinadas por meio do ensaio de fluorescências de raios-x (FRX), estão representadas na Tabela 05.

Tabela 05 - Composição química

\begin{tabular}{lccc}
\hline ElementolMaterial & GC (\% massa) & GCR (\% massa) & RGR (\% massa) \\
\hline $\mathbf{C a O}$ & 70,22 & 69,97 & 68,19 \\
\hline $\mathbf{S O}_{\mathbf{3}}$ & 28,04 & 27,85 & 27,10 \\
\hline $\mathbf{S i O}$ & 0,56 & 0,56 & 2,28 \\
\hline $\mathbf{S r}$ & 0,39 & 0,67 & 0,43 \\
\hline $\mathbf{F e}_{2} \mathbf{O}_{3}$ & 0,38 & 0,44 & 1,09 \\
\hline $\mathbf{A l}_{\mathbf{2}} \mathbf{O}_{\mathbf{3}}$ & 0,26 & 0,00 & 0,53 \\
\hline $\mathbf{K}$ & 0,14 & 0,00 & 0,30 \\
\hline $\mathbf{R h}$ & 0,00 & 0,46 & 0,00 \\
\hline $\mathbf{P}$ & 0,00 & 0,06 & 0,08 \\
\hline
\end{tabular}

Fonte: Os autores

Nota-se que as composições químicas dos gessos estudados são bastante similares contudo percebe-se que o RGR possui um teor um pouco maior de sílica $\left(\mathrm{SiO}_{2}\right)$ e óxido de ferro $\left(\mathrm{Fe}_{2} \mathrm{O}_{3}\right)$ que os demais, possivelmente devido a contaminações. A quantidade de minerais silicosos pode contribuir para redução de resistência mecânica. Observa-se também que todos os gessos apresentaram uma quantidade de $\mathrm{SO}_{3}$ (anidrido sulfúrico) inferior ao valor mínimo exigido pela NBR 13207:1994 (mínimo 53,0\%) para ser considerado como gesso de construção civil.

Os resultados de tempo de pega dos gessos apontam que o RGR tem o início e o fim da pega muito rápida, podendo ser devido à presença de minerais solúveis em água (como halita e silvita) que afetam as propriedades da pasta fresca, como tempo de pega (John e Cincotto, 2010). Os demais gessos reciclados também não atendem às exigências normativas para serem considerados gesso de construção civil e nem de gesso de fundição, que têm tempo de pega menor.

Tabela 06 - Valores de tempo de pega

\begin{tabular}{|l|l|l|l|}
\hline TEMPO DE PEGA & GC & GCR & RGR \\
\hline Início de pega & $13^{\prime} 25^{\prime \prime}$ & $05^{\prime} 50^{\prime \prime}$ & $00^{\prime} 20^{\prime \prime}$ \\
\hline Fim de pega & $22^{\prime} 17^{\prime \prime}$ & $09^{\prime} 20^{\prime \prime}$ & $01^{\prime} 10^{\prime \prime}$ \\
\hline
\end{tabular}

Fonte: Os autores

Com relação à dureza, todos os gessos reciclados estudados apresentaram valores menores que os exigidos por norma, conforme apresentado no Gráfico 02.

O gráfico 03 ilustra os valores médios das resistências à compressão obtidos dos corposde-prova de gesso. Pode-se confirmar as características inferiores dos gessos reciclados, no entanto nem mesmo o $\mathrm{GC}$ atendeu ao requisito da norma quanto à resistência à compressão mínima, embora tenha alcançado alcançando valores próximos aos da norma. O GCR apresentou valores mais altos que o RGR. A baixa resistência à compressão do RGR pode ser devido à existência de vazios na sua estrutura pela dificuldade de moldagem dos corpos-de-prova uma vez que o tempo de pega foi pequeno, podendo ter havido insuficiência de energia de compactação Outra hipótese é por terem sido rompidos no sexto dia e ainda apresentarem umidade, pois não foi 
encontrado em literatura período ideal para determinação da resistência à compressão. A relação água/gesso foi 0,5 .

\section{Gráfico 02 - Valores da dureza dos materiais}

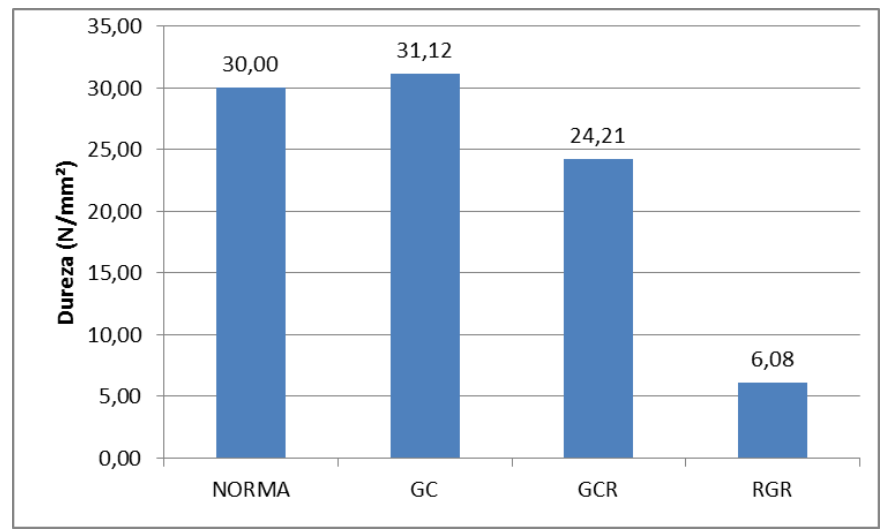

Fonte: Os autores

\section{Gráfico 03 - Resistência à compressão}

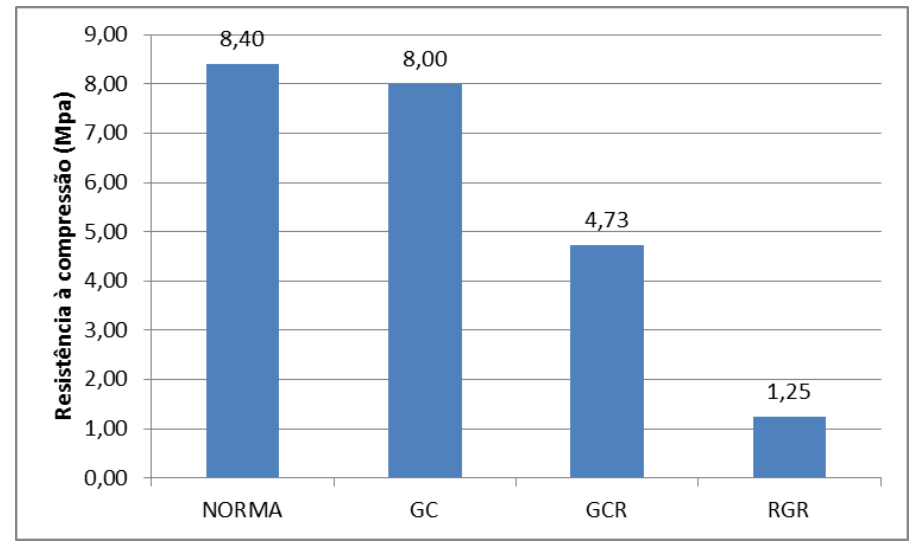

Fonte: Os autores

\section{CONCLUSÕES}

A análise das características dos gessos reciclados (GCR e RGR) e do gesso comercial utilizado como referência indica que seguindo estes métodos para reciclagem do gesso, o GCR e RGR não atendem satisfatoriamente nenhuma das exigências físicas e químicas do pó de gesso, físicas da pasta e mecânica do gesso enrijecido para classificálos como gesso de construção civil pela NBR 13207 (ABNT, 1994) pois estes apresentaram massa unitária inferior à mínima fixada pela norma, quantidade de $\mathrm{SO}_{3}$ (anidrido sulfúrico) inferior à mínima fixada pela norma, tempo de pega fora dos intervalos de classificação como revestimento ou fundição estabelecidos pela norma, dureza e resistência à compressão inferiores ao mínimo exigido por norma.

Apesar do GCR não apresentar nenhuma impureza, este apresentou alguns valores próximos aos da norma, contudo ainda abaixo, não podendo ser considerado como gesso de construção civil. Vale salientar que alguns resultados do GC utilizado estão também abaixo dos padrões exigidos por norma, nos levando a refletir sobre o controle de qualidade dos produtos no mercado. 


\section{AGRADECIMENTOS}

À CAPES pela bolsa e ao GPMATE pelo apoio recebido na execução dos ensaios.

\section{REFERÊNCIAS}

AGOPYAN, A. K.; CARDOSO, F. A; JOHN, V. M.; CARBONI, C. Análise Reológica de Pastas para Revestimentos à Base de Gesso por Squeeze-Flow. VI Simpósio Brasileiro de Tecnologia de Argamassas - I International Syposium on Mortars Technology. Florianópolis, 2005.

AGOPYAN, V. et al. Alternativas para redução de desperdício de materiais nos canteiros de obras. São Paulo, FINEP, ITQC, Escola Politécnica da USP, 1998.

ASSOCIAÇÃO BRASILEIRA DE NORMAS TÉCNICAS (ABNT). NBR 12127: Gesso para construção - Determinação das propriedades físicas do pó - Método de ensaio. Rio de Janeiro, 1991.

NBR 12128: Gesso para construção - Determinação das propriedades físicas da pasta Método de ensaio. Rio de Janeiro, 1991.

. NBR 12129: Gesso para construção - Determinação das propriedades mecânicas -

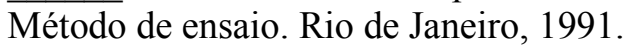

NBR 13207: Gesso para construção civil - Especificação. Rio de Janeiro, 1994.

BARDELLA, P. S.; CAMARINI, G. Calor de hidratação de gesso reciclado para construção. In: CONGRESSO BRASILEIRO DE ENGENHARIA E CIÊNCIAS DOS MATERIAIS, 16., 2004, São Paulo. Anais... São Paulo: Cbecimat, 2004. p. 1-18.

BARDELLA, P. S.; CAMARINI, G. Propriedades de gesso reciclado para utilização na construção civil. In: CONGRESSO BRASILEIRO DE ENGENHARIA E CIÊNCIAS DOS MATERIAIS, 17., 2006, São Paulo. Anais... São Paulo: CBECIMAT, 2006. p. 1-12.

BAUER. L. A. F. Materiais de Construção. 5 ed. Rio de Janeiro: LTC, 2000.

BRASIL. CONAMA - Conselho Nacional do Meio Ambiente. Resolução no 431. 2011.

CAMPBELL, S. L. by Example Walls and Cieling. Disponível em:

http://www.wconline.com/CDA/ArticleInformation/features/BNP_Features_Item/0,3299,937 61,00.html Acessado em 17/07/13.

CARVALHO, M. T. M. Estudo dos resíduos de gesso e suas aplicações na construção civil, 2005. 228 p. Dissertação (Mestrado em Engenharia Civil) - Universidade Federal de Goiás, Goiânia, 2005.

CAVALCANTI, L. B. Determinação de condições operacionais adequadas na desidratação do minério de gipsita para obtenção de um gesso beta reciclável, 2006. 87 p. Dissertação (Mestrado em Engenharia Civil) - Universidade Católica de Pernambuco, Recife, 2006.

CIWMB (California Integrated Waste Management Board) Drywall Recycling. Disponível em: http://www.ciwmb.ca.gov/ConDemo/factsheets/Drywall.htm\#Economics Acesso em: 17 jul. 2013.

JOHN, V. M., CINCOTTO, M. A. Gesso de Construção Civil. In: Materiais de Construção Civil e Engenharia de Materiais. 2 ed. São Paulo: IBRACON, 2010.

MELO, D. C. P. Estudo teórico-experimental da desidratação da gipsita para obtenção de um gesso beta reciclável, 2008. 83 p. Dissertação (Mestrado) - Programa de Pós-Graduação em Engenharia Química, Departamento de Centro de Tecnologia e Geociências, Universidade Federal de Pernambuco, Recife, 2008. 
MONÇÃO JUNIOR, A. R. Otimização das condições experimentais na desidratação da gipsita para obtenção de um gesso beta reciclável, 2008. 66 p. Dissertação (Mestrado em Engenharia Civil) - Universidade Católica de Pernambuco, Recife, 2008.

NIGLIO, F.; CAMARINI, G. Viabilização técnica e econômica da reciclagem do gesso. Campinas: FEC-Unicamp, 2004. 19 p. Relatório Técnico de Iniciação Científica.

NITA, C. et al. Estudo da reciclagem do gesso de construção. In: CONFERÊNCIA LATINOAMERICANA DE CONSTRUÇÃO SUSTENTÁVEL, 1., ENCONTRO NACIONAL DE TECNOLOGIA DO AMBIENTE CONSTRUÍDO, 10., 2004, São Paulo. Anais... . São Paulo: ENTAC, 2004. p. 1-10.

PINHEIRO, S. M. de M. Gesso reciclado: avaliação das propriedades para uso em componentes. 2011.304 p. Tese (Doutorado em Engenharia Civil) - Faculdade de Engenharia Civil, Universidade Estadual de Campinas, Campinas, 2011.

ROQUE, J. A., LIMA, M. M. T. M., CAMARINI, G. Características Químicas e Propriedades Físicas e Mecânicas do gesso reciclado calcinado a $200^{\circ} \mathrm{C}$. In: Congresso Brasileiro de Cerâmica, 49, 2005, São Pedro. Anais... São Pedro, 2005. 